\title{
The Evolution of Heavy Elements in Dwarf Spheroidal Galaxies
}

\author{
Gustavo A. Lanfranchi ${ }^{1}$, Francesca Matteucci ${ }^{2}$ \\ and Gabriele Cescutti ${ }^{2}$ \\ ${ }^{1}$ IAG - USP, R. do Matão 1226, Cidade Universitária, 05508-900 São Paulo, SP, Brazil \\ email: glanfran@usp.br \\ ${ }^{2}$ Dipartimento di Astronomia-Universitá di Trieste, Via G. B. Tiepolo 11, 34131 Trieste, Italy \\ email: matteucci@ts.astro.it, cescutti@ts.astro.it
}

\begin{abstract}
We follow the evolution of barium and europium in four Local Group Dwarf Spheroidal Galaxies by means of a detailed chemical evolution model. The model adopts up to date nucleosynthesis and takes into account the role played by supernovae of different types (II, Ia). By assuming that barium is produced in low mass AGB stars by s-process but also in massive stars (in the mass range 10-30 $M_{\odot}$ ) by r-process and that europium is a pure r-process element synthesized in massive stars in the same range of masses $\left(10-30 M_{\odot}\right)$, we are able to reproduce the observed $[\mathrm{Ba} / \mathrm{Fe}]$ and $[\mathrm{Eu} / \mathrm{Fe}]$ as functions of $[\mathrm{Fe} / \mathrm{H}]$ in all four galaxies studied. We confirm also the important role played by the very low star formation efficiencies $\left(\nu=0.005-0.5 \mathrm{Gyr}^{-1}\right)$ and by the intense galactic winds $\left(w_{i}=6-13\right)$ in the evolution of these galaxies.
\end{abstract}

Keywords. Galaxies: evolution, galaxies: dwarf, galaxies: abundances

\section{The models}

We use the chemical evolution model for dSphs of Lanfranchi \& Matteucci (2003, 2004), in which the dSph galaxies are characterised by one or two long episodes of star formation with low efficiencies and by the occurrence of very intense galactic winds. The nucleosynthesis prescriptions adopt the same yields as in Lanfranchi et al. (2005). For europium it was assumed a r-process production taking place in massive stars in a large range of masses $\left(10-30 M_{\odot}\right)$, while for barium, it was assumed a dominant sprocess occurring in low and intermediate massive stars (LIMS) and a low fraction being produced in massive stars (in the mass range 10-30 $M_{\odot}$ ) through the r-process. The yields from LIMS are taken from Busso et al. (2001). The predictions of the models are compared to the observational data from Carina, Draco, Sculptor and Ursa Minor taken from Shetrone et al. (2001, 2003), Sadakane et al. (2004), Venn et al. (2004) and Geisler et al. (2005).

\section{The evolution of $\mathrm{Ba}$ and $\mathrm{Eu}$}

\subsection{The $[\mathrm{Ba} / \mathrm{Fe}]$ ratio}

The $[\mathrm{Ba} / \mathrm{Fe}]$ pattern is explained by the different contributions to the production of $\mathrm{Ba}$ and by the the effect of the galactic winds on the SFR. The r-processed Ba is more important at early stages of galactic evolution and is responsible for the high values observed at low $[\mathrm{Fe} / \mathrm{H}]$ and to maintain the predicted $[\mathrm{Ba} / \mathrm{Fe}]$ almost constant at suprasolar values at intermediate metallicities. The s-processed Ba produced in LIMS becomes important later, at high metallicities $([\mathrm{Fe} / \mathrm{H}]>-1.9 \mathrm{dex})$, close to the ones when the wind develops. As a consequence, they are more important in avoiding the decrease of $[\mathrm{Ba} / \mathrm{Fe}]$ 

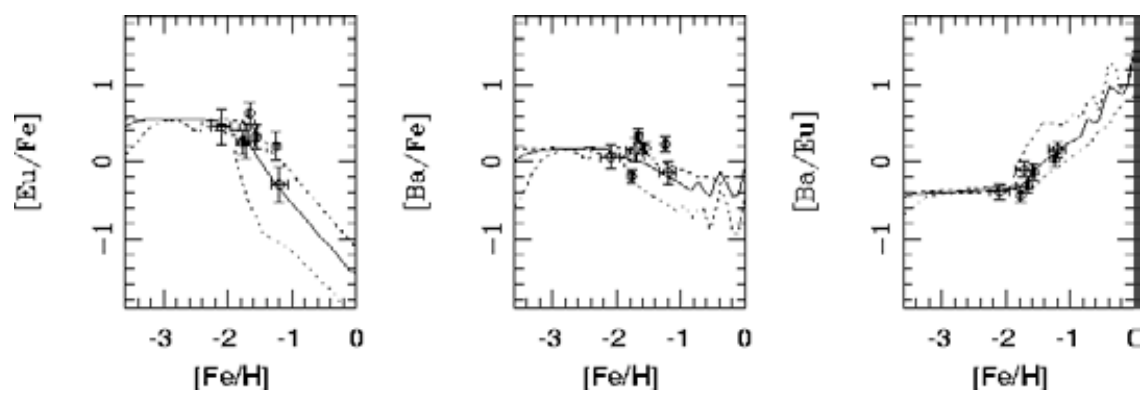

Figure 1. The predicted $\mathrm{Ba}$ and $\mathrm{Eu}$ in Sculptor dSph galaxy compared to the observed data. The solid line represents the best model $\left(\nu=0.2 \mathrm{Gyr}^{-1}, \mathrm{w}_{i}=13\right)$ and the dotted lines the lower $\left(\nu=0.05 \mathrm{Gyr}^{-1}\right)$ and upper $\left(\nu=0.5 \mathrm{Gyr}^{-1}\right)$ limits for the SF efficiency.

to be even more intense and in reproducing the data of the stars which appear soon after the onset of the wind.

\subsection{The $[\mathrm{Eu} / \mathrm{Fe}]$ ratio}

The observed $[\mathrm{Eu} / \mathrm{Fe}]$ ratio is very well reproduced by the models for all four dSph galaxies. At the early stages of evolution the major contributor to the ISM enrichment are the massive stars, which produce much more Eu than $\mathrm{Fe}$, giving rise to a high [Eu/Fe] at low $[\mathrm{Fe} / \mathrm{H}](<-2.0 \mathrm{dex})$. When the first SNe Ia start exploding the enrichment of Fe becomes much more important and the $[\mathrm{Eu} / \mathrm{Fe}]$ starts decreasing. With the energy released by these explosions the galactic winds are triggered and, since they remove a large fraction of the gas reservoir which fuels the SF, the SFR drops down considerably. As a consequence, the enrichment of $\mathrm{Eu}$ is almost halted but the one of Fe continues for a long time-scale giving rise to very low values of $[\mathrm{Eu} / \mathrm{Fe}]$, as observed.

\subsection{The $[B a / E u]$ ratio}

The sub-solar "plateau" seen in the $[\mathrm{Ba} / \mathrm{Eu}]$ vs. $[\mathrm{Fe} / \mathrm{H}]$ plot at low metallicities is caused by the injection into the ISM of $\mathrm{Ba}$ and $\mathrm{Eu}$ by massive stars, in different fractions: $\mathrm{Eu}$ is assumed to be a pure r-process element, whereas the fraction of r-processed $\mathrm{Ba}$ is low. This gives rise to a sub-solar $[\mathrm{Ba} / \mathrm{Eu}]$. When the first SNe Ia explode and the wind develops, there is an abrupt change and the $[\mathrm{Ba} / \mathrm{Eu}]$ suffers an intense increase, due to the injection in the ISM of Ba by LIMS and to the decrease in the SFR. With a very low SFR, the production of $\mathrm{Eu}$ is almost halted, but the one of $\mathrm{Ba}$ continues since the LIMS have a long lifetime (some Gyrs). In this sense, $[\mathrm{Ba} / \mathrm{Fe}]$ increases considerably, as observed in the dSph stars.

\section{Conclusions}

The nucleosynthesis prescriptions adopted in this work are the same which allow a chemical evolution model for our Galaxy to reproduce very well the $[\mathrm{Ba} / \mathrm{Fe}],[\mathrm{Eu} / \mathrm{Fe}]$ and $[\mathrm{Ba} / \mathrm{Eu}]$ trends observed in metal-poor stars (Cescutti et al. 2005). This agreement coupled to the one achieved here strongly suggests that the our assumptions regarding the formation and production of $\mathrm{Ba}$ and $\mathrm{Eu}$ are quite reasonable.

\section{References}

Busso M., Gallino R., Lambert D.L., Travaglio C., \& Smith V.V. 2001, ApJ 557, 802

Cescutti, G., François, P., Matteucci, F., Cayrel, R., Spite, M., \& Spite, F. 2005, A\& $A$ submitted 
Geisler D., Smith V.V., Wallerstein G., Gonzalez G., \& Charbonnel C. 2005, AJ, 129, 1428

Lanfranchi, G. \& Matteucci, F. 2003, MNRAS 345, 71

Lanfranchi, G. \& Matteucci, F. 2004, MNRAS 351, 1338

Lanfranchi, G., Matteucci, F., \& Cescutti, G. 2005, MNRAS submitted

Sadakane K., Arimoto N., Ikuta C., Aoki W., Jablonka P., \& Tajitsu A. 2004, PASJ, 56, 1041

Shetrone M., Côté P., \& Sargent W.L.W. 2001, ApJ, 548, 59

Shetrone M., Venn K.A., Tolstoy E., \& Primas F. 2003, AJ, 125, 684

Venn K. A., Irwin M., Shetrone M.D., Tout, C.A., Hill V., \& Tolstoy E. 2004, AJ, 128, 1177 


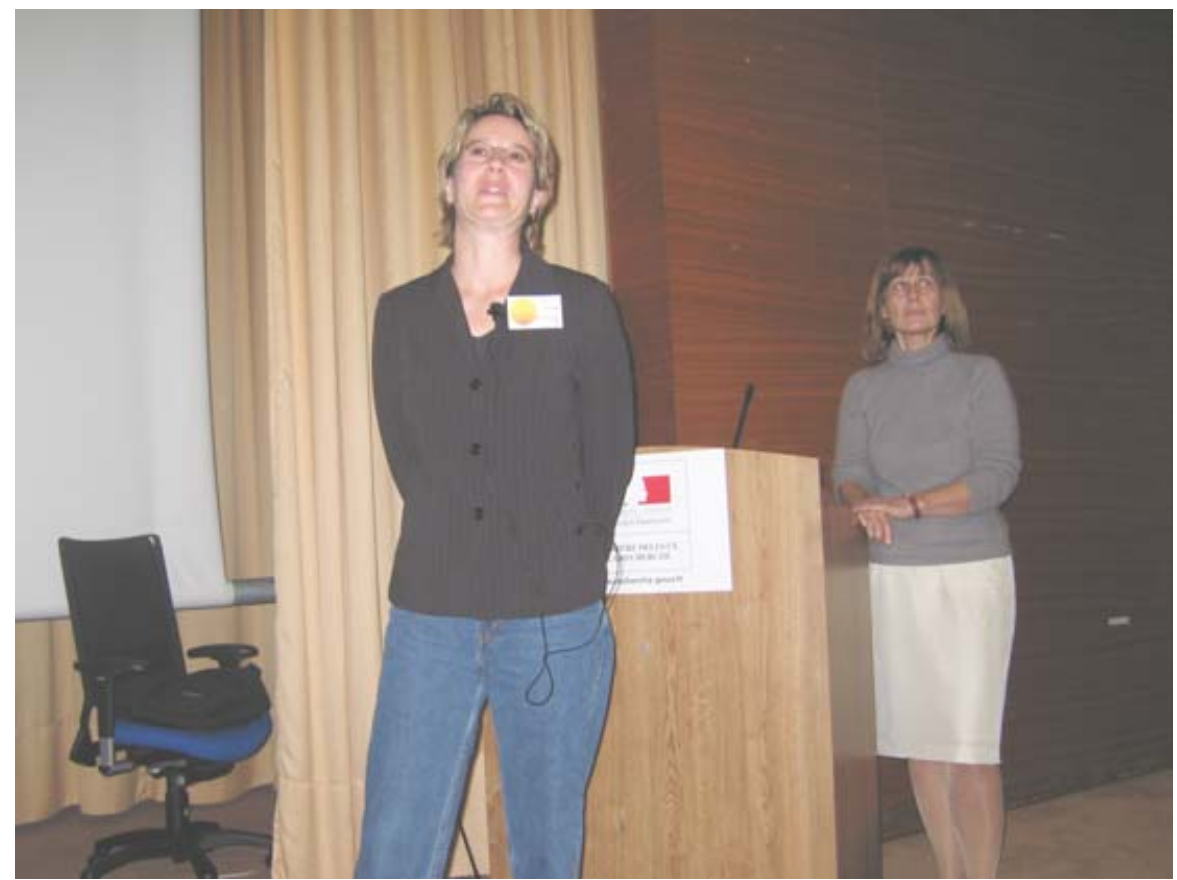

Kim Venn talking about the Sculptor dSph galaxy, chaired by Beatriz Barbuy.

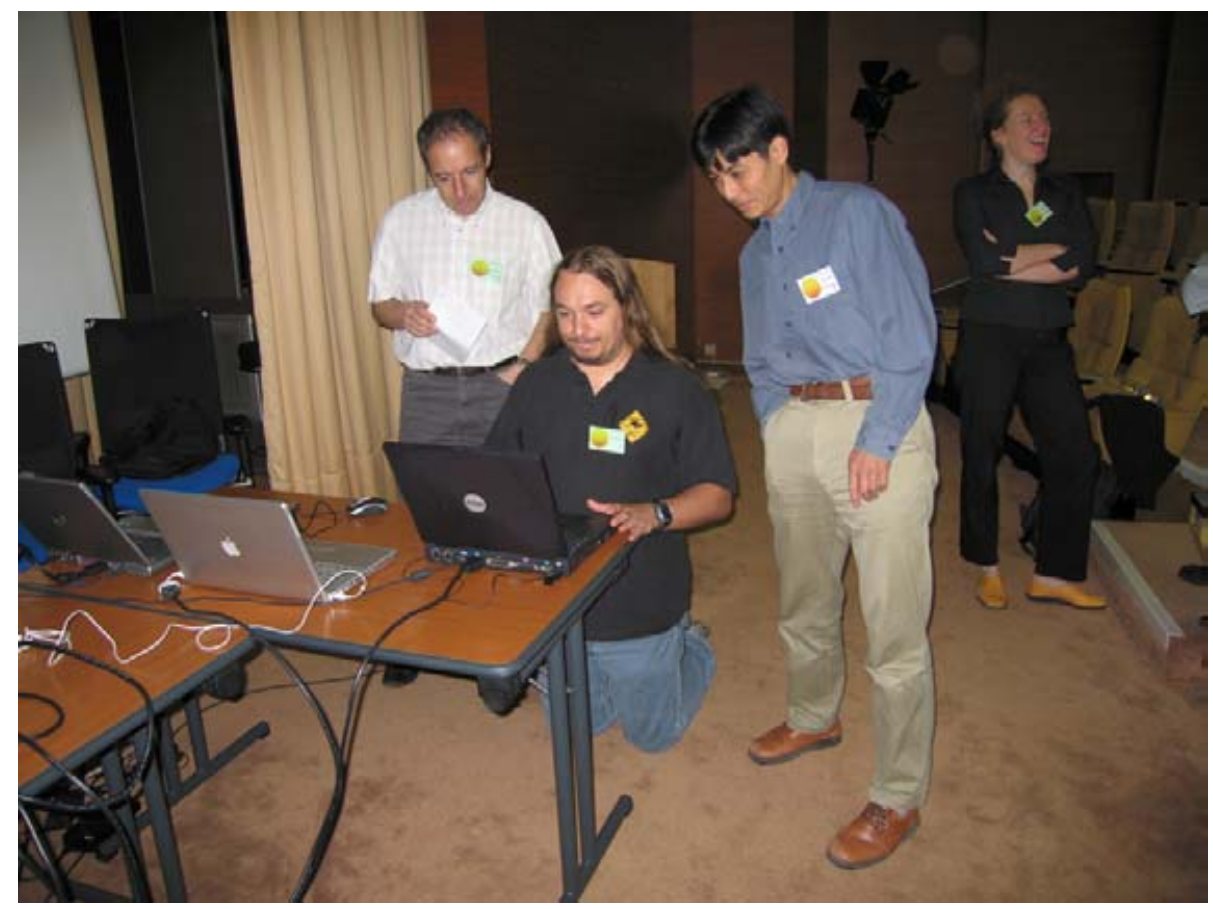

The LOC technical team (Patrick François, and Eric Depagne kneeling down) preparing for the next session's presentations under the eyes of Wako Aoki. 